\title{
SINGULARIDAD DE UN SISTEMA ACOPLADO ${ }^{(1)}$
}

\author{
Luis Enrique Carrillo Díaz \\ E-mail: d230112@unmsm.edu.pe \\ Icarrillo@latinmail.com \\ Teófanes Quispe Méndez \\ Universidad Nacional Mayor de San Marcos
}

\section{RESUMEN}

Condiciones suficientes son dadas de modo que soluciones del problema de valor inicial en la frontera para un sistema acoplado de Kirchhoff no existan para todo $t>0$.

Palabra Claves: Ecuaciones hiperbólicas, soluciones locales débiles, "blow-up" de soluciones.

\section{INTRODUCIÓN}

En éste trabajo estamos interesados en estudiar las propiedades de "Blowing-up" de las soluciones del sistema acoplado siguiente :

$$
\mid \begin{array}{lc}
u_{u}-M\left(\|u(t)\|^{2}\right) \Delta u=\gamma|u|^{\rho+2}|u|^{\rho} u & \text { en } Q \\
v_{u}-M\left(\|v(t)\|^{2}\right) \Delta v=\gamma|u|^{\rho+2}|v|^{\rho} v & \text { en } Q \\
u(0)=u_{0}, u_{t}(0)=u_{1} & \text { en } \Omega \\
v(0)=v_{0}, v_{t}(0)=v_{1} & \text { en } \Omega
\end{array}
$$

Aquí $Q$ es el cilindro $\Omega x$ ] $0, T$ [ para $T>0$ un número real que ha sido escogido arbitrariamente y luego se ha fijado. El conjunto $\Omega$ es un abierto acotado de $\mathfrak{R}^{n}(n=1,2,3)$, y los números $\gamma$ y $\rho$ son reales con restricciones especificadas posteriormente.

La función $\mathrm{M}$ es una función de clase $C^{1}([0,+\infty)$, dada por

$$
\mathrm{M}(s)=\alpha+(\rho+2) \beta S^{\rho+1}
$$

${ }^{(1)}$ Este trabajo fue financiado por el Consejo Superior de Investigaciones de la UNMSM. Proyecto $\mathrm{N}^{\circ} 81401011$ 
Es importante notar que cuando $v$ coincide con $u$, entonces $\left(^{*}\right)$ se reduce a la ecuación.

$$
u_{t t}-M\left(\|u\|^{2}\right) \Delta u=\gamma u^{3}
$$

la cual ha sido estudiada como un caso general del problema de Klein-Gordon. El sistema (*) ha sido estudiado por Milla Miranda-Medeiros [6] para el caso $M \equiv 1$ con el parámetro $\rho>-1$ para $n=1,2$ y $-1<p \leq \frac{2 n}{n-2}$., si $n \geq 3$.

En dicho trabajo ellos obtienen solución global débil usando el método del Pozo de Potencial introducido por Sattinger [8] y Lions [5]. En el caso en que $\mathrm{M} \equiv 1$ y $\rho \equiv 0$ se tiene el modelo matemático de la interacción de dos partículas con cargas electromagnéticas; claramente el modelo especifico es con el término $\alpha^{2} u \quad y \quad \alpha^{2} v$ sumados en el primer miembro.

Inspirados en el método del Pozo de Potencial, Ikehata-Okazawa [3] han ideado un método que consiste en colocar los datos iniciales en un conjunto llamado de Inestabilidad, para de ese modo obtener soluciones del sistema $(*)$ las cuales desarrollen una singularidad en tiempo finito. Esta singularidad es obtenida en el sentido de la norma. En algunas oportunidades se obtiene la singularidad de la misma solución, pero las técnicas empleadas son bien elaboradas. Como una observación adicional es interesante notar que cuando $v=u$, el sistema $\left(^{*}\right)$ se reduce a la ecuación.

$$
u_{t t}-M\left(\|u(t)\|^{2}\right) \Delta u=|u|^{2} \rho+2 u
$$

ó en forma equivalente a :

$$
u_{t t}-\mathrm{M}\left(\|u(t)\|^{2}\right) \Delta u=\left(|u|^{2}\right)^{\rho+1} u
$$

Cuando $M \equiv 1$, la ecuación (2) es un caso particular de la ecuación

$$
u_{t t}-\Delta u+\alpha^{2} u \equiv F\left(|u|^{2}\right) u
$$

la cual tiene aplicación en el área de óptica no lineal, Física del plasma, Mecánica de Fluídos, etc. 
la cual tiene aplicación en el área de óptica no lineal, Física del plasma, Mecánica de Fluídos, etc.

La generalización natural de $\left(^{*}\right)$ es el sistema

$$
\left\{\begin{array}{l}
u_{t t}-\mathrm{M}\left(\|u(t)\|^{2}\right) \Delta u=F\left(|u|^{2},|v|^{2}\right) u \\
v_{t t}-\mathrm{M}\left(\|v(t)\|^{2}\right) \Delta v=G\left(|u|^{2},|v|^{2}\right) v
\end{array}\right.
$$

para F y G funciones "adecuadas".

\section{EXISTENCIA LOCAL}

Haciendo uso del método de Galerkin y de estimativas ingeniosas inspiradas en Tartar [9] se obtiene la existencia de la solución local $[u, v]$ del sistema $\left(^{*}\right)$.

\subsection{TEOREMA (EXISTENCIA LOCAL)}

Sea $\mathrm{M} \in C^{1}([0,+\infty D), \mathrm{M}(s) \geq \alpha>0$ para $s \in[0,+\infty[, \gamma>0$ para $n=1,2$ y $\rho=0$ para $n=3$. Si $u_{0}, v_{0} \in H^{2}(\Omega) \cap H_{0}^{1}(\Omega)$ y $u_{1}, v_{1} \in H_{0}^{1}(\Omega)$, entonces existe $T_{0} \in \Re, 0<T_{0} \leq T$ y funciones $u, v: \Omega x\left[0, T_{0}\right] \rightarrow \Re$ tales que:

$$
\begin{aligned}
& u . v \in L^{\infty}\left(0, T_{0} ; H^{2}(\Omega) \cap H_{0}^{1}(\Omega)\right) \\
& u^{\prime} v^{\prime} \in L^{\infty}\left(0, T_{0} ; H_{0}^{1}(\Omega)\right) \\
& u^{\prime \prime}, v^{\prime \prime} \in L^{2}\left(0, T_{0} ; L^{2}(\Omega)\right)
\end{aligned}
$$

y $[u, v]$ es solución débil del problema $\left(^{*}\right)$ en $\Omega x\left[0, T_{0}\right]$.

\section{DEMOSTRACIÓN}

Sea $\left\{w_{v}\right\}$ una base Hilbertiana de $H_{0}^{1}(\Omega) \cap H^{2}(\Omega)$ formada por las funciones propias del operador $-\Delta$; es decir los $w_{v}$ satisfacen

$$
\begin{array}{ll}
-\Delta w_{v}=\lambda_{v} w_{v} & \text { en } \Omega \\
w_{v}=0 & \text { en } \Gamma
\end{array}
$$


Sea $V_{m}=\left[w_{1}, \ldots ., w_{m}\right]$ el subespacio generado por los primeros $m$ vectores $w_{1}, \ldots, w_{m}$ de $\left\{w_{v}\right\}$. Consideremos

$$
u_{m}(t)=\sum_{j=1}^{m} g_{j m}(t) w j, v_{m}(t)=\sum_{j=1}^{m} h_{j m}(t) w_{j}
$$

soluciones aproximadas en $V_{m}$ del problema $(*)$. Las funciones $g_{j m}(t)$ y $h_{j m}(t)$; $j=1,2, \ldots ., m$ son determinadas por el siguiente problema de EDO para $j=1,2$, ...., $m$ :

$$
\text { (P.A) } \begin{aligned}
& \left(u_{m}^{\prime \prime}(t), w_{j}\right)+M\left(\left|u_{m}(t)\right|^{2}\right) a\left(u_{m}(t), w_{j}\right)=\gamma\left(v_{m}(t)^{\rho+2}\left|u_{m}\right|^{\rho} u_{m}(t), w_{j}\right) \\
& \left(v_{m}^{\prime \prime}(t), w_{j}\right)+M\left(\left.v_{m}(t)\right|^{2}\right) a\left(v_{m}(t), w_{j}\right)=\gamma\left(u_{m}(t)^{\rho+2} v_{m}{ }^{\rho} v_{m}(t), w_{j}\right) \\
& u_{m}^{\prime}(0)=u_{1 m} \rightarrow u_{0} \text { fuerte en } H_{0}^{1}(\Omega) \cap H^{2}(\Omega) \\
& v_{m}(0)=v_{o m} \rightarrow v_{0} \text { fuerte en } H_{0}^{1}(\Omega) \\
& v_{m}^{\prime}(0)=v_{m} \rightarrow v_{1} \text { fuerte en } H_{0}^{1}(\Omega) \cap H^{2}(\Omega)
\end{aligned}
$$

Haciendo uso del Teorema de Caratheodory se garantiza la existencia de una solución del problema aproximado en el intervalo $\left[0, t_{m}\right]$ donde $t_{m}>0$ y $t_{m}<T$.

Con algunas estimativas extenderemos la solución $[u, v]$ a un intervalo $\left[0, T_{0}\right] \subset[0, T]$, con $T_{0}$ independiente de $\mathrm{m}$.

\section{1era. Estimativa a-priori}

Multiplicando las dos primeras ecuaciones del problema aproximado (P.A) por $g_{j m}^{\prime}(t)$ y $h_{j m}^{\prime}(t)$ respectivamente y sumando $j$ desde 1 hasta $m$ y luego sumando miembro a miembro se obtiene :

$$
\begin{gathered}
\frac{d}{d t}\left[\left|u_{m}^{\prime}(t)\right|^{z}+\left|v_{m}^{\prime}(t)\right|^{2}+M\left(\|\left. u_{m}(t)\right|^{2}\right)+M\left(\|\left. v_{m}(t)\right|^{2}\right)\right]= \\
2 \gamma\left[\left(\mid v_{m}(t)^{\rho+2} u_{m}(t)^{\rho} u_{m}(t), u_{m}^{i}(t)\right)+\left(u_{m}(t)^{\rho+2} \mid v_{m}(t)^{\rho} v_{m}(t), v_{m}^{i}(t)\right)\right.
\end{gathered}
$$

donde $\quad \not \square(s)=\int_{0}^{\varsigma} M(\xi) d \xi$ 
Denotando por $\mu_{m}(t)$ al segundo miembro de (4) y aplicando luego las desigualdades de Cauchy-Schwartz, Holder

$$
\begin{aligned}
\mu_{m}(t) & \leq 2 \gamma\left[\left.\left.v_{m}(t)\right|_{P(\rho+2)} ^{\rho+2} u_{m}(t)\right|_{q(\rho+1)} ^{\rho+1} \mid u_{m}^{\prime}(t)\right. \\
+ & u_{m}(t)_{p(\rho+2)}^{\rho+2}\left|v_{m}(t)_{q(\rho+1)}^{\rho+1}\right| v_{m}^{\prime}(t) \mid
\end{aligned}
$$

donde $\frac{1}{P}+\frac{1}{q}=1 / 2$.

Haciendo uso de las inmersiones de Sobolev y de las inmersiones de los espacios $L^{P(\rho+2)}(\Omega)$ y $L^{q(\rho+2)}(\Omega)$ en $H_{0}^{1}(\Omega)$

Se obtiene

$$
\mu_{m}(t) \leq C\left[\left\|\left.v_{m}(t)\right|^{\rho+2}\right\| u_{m}(t)^{\rho+1}\left|u_{m}^{\prime}(t)+\left\|u_{m}(t)\right\|^{\rho+2} \| v_{m}(t)\right|^{\rho+1} v_{m}^{\prime 1}(t)\right]
$$

$$
C>0 \text { (constante) }
$$

Sea $\varphi_{m}(t)$ definida por

$$
\varphi_{m}(t)=\mid u_{m}^{\prime}(t)^{2}+v_{m}^{\prime}(t)^{2}+\tilde{M}\left(\| u_{m}(t)^{2}\right)+\tilde{M}\left(v_{m}(t)^{2}\right)
$$

(6)

de aquí obtenemos

$$
\begin{array}{ll}
\mid u_{m}^{\prime}(t) \leq \varphi_{m}^{1 / 2}(t), & v_{m}^{\prime}(t) \leq \varphi_{m}^{1 / 2}(t) \\
u_{m}(t) \mid \leq \frac{\varphi_{m}^{1 / 2}(t)}{\sqrt{\alpha}}, & v_{m}(t) \mid \leq \frac{\varphi_{m}^{1 / 2}(t)}{\sqrt{\alpha}}
\end{array}
$$

Usando éstas desigualdades tenemos

$$
\varphi_{m}^{\prime}(t) \leq C_{2}\left[\varphi_{m}^{\rho+2}(t)\right], \quad C_{2}>0 \quad \text { Constante }
$$

de donde

$$
\varphi_{m}^{\prime}(t) \leq C_{3}\left[1+\varphi_{m}^{\rho+1}(t)\right] \quad, \quad C_{3}>0 \quad \text { Constante }
$$


integrando de 0 a t obtenemos :

$$
\varphi_{m}(t) \leq \varphi_{m}(0)+C_{3}\left[T+\int_{0}^{t} \varphi_{m}^{p+1}(s) d s\right]
$$

Como la función $\widetilde{M}(S)$ es continua en $[0, \infty[$ y por las condiciones iniciales del problema aproximado (P.A) se tiene que $\varphi_{m}(0)$ converge a $A=\left|u_{1}\right|^{2}+\left|v_{1}\right|^{2}+M\left(\left\|u_{0}\right\|^{2}\right)+M\left(\left\|v_{0}\right\|^{2}\right)$

Luego para $\varepsilon_{1}>0$ se tiene

$$
\varphi_{m}(0) \leq \varepsilon_{1}+A
$$

de (7) y (8) se tiene que

$$
\varphi_{m}(t) \leq K+\int_{0}^{t} \varphi_{m}^{\rho+2}(s) d s
$$

donde $K>0$ es una constante que depende de los datos iniciales; luego por Gronwall se tiene que existe $T_{1}>0$ y $K_{1}$

$$
\left|u_{m}^{\prime}(t)\right|^{2}+\left|v_{m}^{\prime}(t)^{2}+\left\|\left.u_{m}(t)\right|^{2}+\right\| v_{m}(t)\right|^{2} \leq K_{1}
$$

para $t \in\left[0, T_{1}\right]$.

2da. Estimativa a priori: Multiplicando en las dos primeras ecuaciones del problema aproximado (P.A) por $g_{j m}^{\prime}(t) \lambda j \mathrm{y} h_{j m}^{\prime}(t) \lambda j$ respectivamente, luego sumando según $j$ de 1 hasta $m$, y adicionando miembro a miembro se obtiene

$$
\begin{aligned}
& \frac{d}{d t}\left[\left\|u_{m}^{\prime}(t)\right\|^{2}+\|\left. u_{m}^{\prime}(t)\right|^{2}+M\left(\|\left. u_{m}(t)\right|^{2}\right) \mid \Delta v_{m}(t)^{2}\right] \\
& =2 \gamma\left[\left(\nabla\left(\left.v_{m}(t)\right|^{\rho+2} \mid u_{m}(t)^{\rho} u_{m}(t)\right), \nabla u_{m}^{\prime}(t)\right)\right. \\
& \left.+\left(\nabla\left(\left|u_{m}(t)^{\rho+2}\right| v_{m}(t)^{\rho} v_{m}(t), \nabla v_{m}^{\prime}(t)\right)\right)\right] \\
& +2\left[M ^ { \prime } \left(\|\left. u_{m}(t)\right|^{2} a\left(u_{m}(t), u_{m}^{\prime}(t)\right) \mid \Delta u_{m}(t)^{2}\right.\right. \\
& +M^{\prime}\left(v_{m}(t) \|^{2} a\left(v_{m}(t), v_{m}^{\prime}(t)\right) \mid \Delta v_{m}(t)^{2}\right)
\end{aligned}
$$




\section{Hacemos}

$$
\begin{aligned}
& { }_{m}(t)=\left\|u_{m}^{\prime}(t)\right\|^{2}+\left\|v_{m}^{\prime}(t)\right\|^{2}+M\left(\left\|u_{m}(t)\right\|^{2}\right)+\mid \Delta u_{m}(t) \|^{2} M\left(\left\|v_{m}(t)\right\|^{2}\right) \\
& \left|\Delta v_{m}(t)\right|^{2}
\end{aligned}
$$

Con lo cual de (10) se obtiene después de acotar adecuadamente los sumandos del 2do. miembro:

$$
\varphi_{m}^{\prime}(t) \leq K_{2}\left[1+\psi_{m}^{\rho+3 / 2}(t)\right.
$$

Por argumento similares a los usados en la primera estimativa se llega a verificar que existe $T_{2}>0$ y $K_{3}>0$ tal que:

$$
\left\|u_{m}^{\prime}(t)\right\|^{2}+\left\|v_{m}^{\prime}(t)\right\|^{2}+\left|\Delta u_{m}(t)\right|+\left|\Delta v_{m}(t)\right| \leq K_{3} ; \text { para } t \in\left[o, T_{2}\right]
$$

(OBS: $T_{2}$ depende de los datos iniciales)

\section{3era. Estimativa A-priori:}

Multiplicando ahora en el (P.A) por $g_{j m}^{\prime \prime}(t)$ y $h_{j m}^{\prime \prime}(t)$ en la primera y segunda ecuación respectivamente se obtiene que $\exists K_{4}>0, T_{3}>0$ tal que

$$
\begin{aligned}
& \left.\left|u_{m}^{\prime \prime}(t)^{2}+\right| v_{m}^{\prime \prime}(t)\right|^{2} \leq K_{4} \quad \text { Para } t \in\left[o, T_{3}\right] \\
& \left(T_{3}=\min \left\{T_{1}, T_{2}\right\}\right)
\end{aligned}
$$

Con éstas estimaciones se obtiene el pasaje al límite de forma estándar probándose la existencia de solución local débil.

\section{SINGULARIDAD EN TIEMPO FINITO}

Siguiendo el método empleado en IKEHATA-OKAZAWA [3] combinado con las ideas de ONO [7] basadas en Levine [4] obtenemos "Blow-up" para la solución [u, ]. Esta singularidad en tiempo finito es en el sentido de la norma. 


\subsection{Energía Asociada al Sistema (*) :}

Denotaremos por $V^{1}=H_{0}^{1}(\Omega) x H_{0}^{1}(\Omega), V^{2}=H^{2}(\Omega) x H^{2}(\Omega)$ y $H=L^{2}(\Omega) x L^{2}(\Omega)$ con las normas dadas por

$$
\|[u, v]\|^{2}=u\left\|^{2}+\right\| v \|^{2} \quad\left(\operatorname{Para} V^{1}\right)
$$

y

$$
[u, v]^{2}=u^{2}+v^{2} \quad(\text { Para } H)
$$

Procediendo formalmente, multiplicamos $\left(*_{1}\right)$, y $\left(*_{2}\right)$ por $u$ " y respectivamente, luego integramos sobre $\Omega$ suponiendo que $u=0, \quad=0$ en $\partial \Omega$, luego sumamos miembro a miembro y obtenemos.

$$
\begin{gathered}
\frac{d}{d t}\left\{\frac{1}{2}\left[u^{\prime}(t), v^{\prime}(t)\right]^{2}+\frac{1}{2}\left(M\left(\|\left. u(t)\right|^{2}\right)+M\left(\|\left. v(t)\right|^{2}\right)\right)\right. \\
\left.-\frac{\gamma}{\rho+2} \|\left. u(t) v(t)\right|_{L^{\rho+2}(\Omega)} ^{\rho+2}\right\}=0
\end{gathered}
$$

donde $M(S)=\int_{b}^{s} M(\xi) d \xi$

Definimos la energía del sistema (*) por

$$
\begin{gathered}
E(t)=\frac{1}{2}\left[u^{\prime}(t), v^{\prime}(t)\right]^{2}+\frac{1}{2}\left(M\left(\|u(t)\|^{2}\right)+M\left(\|\left. v(t)\right|^{2}\right)\right) \\
-\frac{\gamma}{\rho+2}\|u(t) v(t)\|_{L^{\rho+2(\Omega)}}^{\rho+2}
\end{gathered}
$$

donde $t \in\left[0, t_{\max }[\right.$.

La energía total $\mathrm{E}(\mathrm{t})$ está dada en términos de la energía cinética $K([u(t), v(t)])$ y potencial $J([u(t), v(t)])$ dados por :

$$
\begin{gathered}
K([u(t), v(t)])=\frac{1}{2}\left[u^{\prime}(t), v^{\prime}(t)\right]^{2} \\
J([u(t), v(t)])=\frac{1}{2}\left(M\left(u(t) \|^{2}\right)+M\left(\|\left. v(t)\right|^{2}\right)\right)-\frac{\gamma}{\rho+2} \|\left. u(t) v(t)\right|_{L^{\rho+2}} ^{\rho+2}
\end{gathered}
$$


Haciendo uso del resultado de inmersión

$$
H_{0}^{1}(\Omega) \rightarrow L^{2(\rho+2)}(\Omega)
$$

Se tiene que el funcional de energía potencial:

$J: H_{0}^{1}(\Omega) x H_{0}^{1}(\Omega) \rightarrow \Re$ dado por $[u, v] \rightarrow J([u, v])$ definido por (18) es "bien definido".

Es en ésta parte de nuestro estudio en que comenzamos a usar ideas del método de Pozo de Potencial el cual es usado para tratar de obtener soluciones globales.

Usaremos la idea del Pozo de Potencial para construir un conjunto de Inestabilidad.

En palabras gráficas el método para obtener singularidad consistirá en no ubicar los datos dentro del Pozo pero si en algún espacio en las cercanías del Pozo.

3.2 Definición.- Al número $d$ se le define por:

$$
d=\operatorname{Inf} .\left\{\operatorname{Sup} . J\left(\lambda[u, v] ;[u, v] \in V^{1} ;[u, v] \neq 0\right)\right.
$$

no olvidemos que estamos trabajando con una función $M(S)=\alpha+(\rho+2) \beta S^{\rho+1}, \alpha>0, \beta \geq 0$; esto es por detalles técnicos a causa del método seguido. Pero es posible trabajar con una función $\mathrm{M}(\mathrm{s})$ más general con hipótesis apropiadas de homogeneidad.

3.3 Lema.- Supongamos que se cumple la relación

$$
\begin{aligned}
& \gamma\{C(2(\rho+2), \Omega)\}^{2(\rho+2)}>(\rho+2) \beta, \\
& \quad \text { entonces } d>0 .
\end{aligned}
$$

(donde $C(2(\rho+2), \Omega)$ es la constante de inmersión de $H_{0}^{1}(\Omega) \rightarrow L^{2(\rho+2)}(\Omega)$ ) 


\section{Demostración}

La demostración sigue el mismo esquema de Carrillo [2].

\section{SINGULARIDAD DE LA SOLUCIÓN}

Sea:

$$
P_{2}=\left\{[u, v] \in V^{1} ; J([u, v])<d,(\rho+2) \beta\left[\|u\|^{2(\rho+2)}+\|v\|^{2(\rho+2)}\right]-2 \gamma\|u v\|_{\rho+2}^{\rho+2}<0\right.
$$

Definimos

$$
V^{*}=\left\{[u, v] \in V^{1} ; J([u, v])<d, \alpha\left\|[u, v]^{2}+(\rho+2) \beta\left[u\left\|^{2(\rho+2)}+\right\| v \|^{2(\rho+2)}\right]<2 \gamma\right\| u v \|_{\rho+2}^{\rho+2}\right.
$$

3.4 Teorema.- Supongamos que (20) se cumpla. Si $[u(t), v(t)]$ es una solución del problema $\left(^{*}\right)$ en $\left[0, t_{\max }\left[\right.\right.$ con datos iniciales $\left[u_{0}, v_{0}\right] \in V^{2} \cap V^{*}$ y $\left[u_{1}, v_{1}\right] \in V^{1}$ satisfaciendo

$$
E(0)<d
$$

entonces $t_{\max }<+\infty$.

Antes de dar un esquema de la demostración observamos que éste teorema nos da una condición de no existencia global tanto para energía inicial $E(0)$ no positiva como para el caso positivo.

Resultados de singularidad en tiempo finito son más frecuentes cuando la energía inicial es negativa ó cero, pero cuando $E(0)>0$ los resultados de blow-up son poco frecuentes.

Daremos algunos lemas que se usan en la demostración del teorema.

3.5 Lema.- $\mathrm{Si}[u(t), v(t)]$ es una solución en las condiciones del teorema en 3.4., entonces $[u(t), v(t)] \in V^{*}$ sobre $\left[0, t_{\max }[\right.$.

3.6 Lema.- Si $[u(t), v(t)]$ está en la condiciones del teorema en 3.4.

Entonces $(\rho+1) \alpha[u,(t), v(t)]^{2}>2(\rho+2) d$ en $\left[0, t_{\max }[\right.$. 
Las demostraciones de los lemas de 3.5 y 3.6 siguen mutatis mutandis a Carrillo [2].

Demostración del Teorema (3.4) Se procede por contradicción

Supongamos que $t_{\max }=+\infty$.

Multiplicando en $\left(*_{1}\right)$ y $\left(*_{2}\right)$ por $u(t)$ y $v(t)$ e integrando sobre $\Omega$, luego sumando miembro a miembro obtenemos :

$\frac{1}{2} \frac{d^{2}}{d t^{2}}\left|[u(t), v(t)]^{2}=(\rho+3)\right|[u(t), v(t)]^{2}+(\rho+1) \alpha \|[u(t), v(t)]^{2}-2(\rho+2) E(0)$

Usando Lema 3.6 se tiene

$$
\frac{d^{2}}{d t^{2}}[u(t), v(t)]^{2}>2(\rho+3)\left[u^{\prime}(t), v^{\prime}(t)\right]^{2}+C_{0}
$$

donde $C_{0}=4(\rho+2)(d-E(0))>0$

Haciendo $P(t)=[u(t), v(t)]^{2}$

obtenemos

$$
P^{\prime}(t)=2\left\{\left(u^{\prime}(t), u(t)\right)+\left(v^{\prime}(t), v(t)\right)\right\}
$$

y de (24) se tiene

$$
P^{\prime \prime}(t)>C_{0}
$$

integrando esta desigualdad de 0 a $t$ tenemos

$$
P^{\prime}(t) \geq 2\left\{\left(u_{1}, u_{0}\right)+\left(v_{1} v_{0}\right)\right\}+C_{0} t
$$

lo cual implica que existe $t_{0}>0$ tal que

$$
\left.P^{\prime}(t)>0 \text { en }\right] t_{0},+\infty[
$$


De (25) y (26) vemos que $\mathrm{P}(\mathrm{t}) \neq 0$ en $] t_{0},+\infty[$.

Ahora multiplicamos (24) por $\mathrm{P}(t)$ y sumamos $\frac{-(\rho+3)}{2}\left(P^{\prime}(t)\right)^{2}$ obteniendo

$$
\begin{gathered}
P^{\prime \prime}(t) P(t)-\frac{(\rho+3)}{2}\left(P^{\prime}(t)^{2}>2(\rho+3)\right)\left\{\left[u^{\prime}(t), v^{\prime}(t)\right)^{2} \mid[u(t), v(t)]^{2}-\left\{\left(u^{\prime}(t), v^{\prime}(t)\right)\left(v^{\prime}(t), v(t)\right)\right\}^{2}\right\}+C_{0} P(t) \\
\left.>C_{0} P(t) e n\right] t_{0},+\infty[
\end{gathered}
$$

En (27) hemos empleados la desigualdad de Cauchy-Schwartz

A continuación hacemos $F(t)=[u(t), v(t)]^{-1}$ para $t>t_{0}$.

Es decir

$F(t)=P^{-1 / 2}(t)$; así derivando obtenemos

$F^{\prime}(t)=\frac{1}{2} P^{-3 / 2}(t) P^{\prime}(t)$

$F^{\prime \prime}(t)=\frac{-1}{2} P^{-5 / 2}(t)\left(P^{\prime \prime}(t) P(t)-\frac{3}{2} P^{2}(t)\right)$

De (27) resulta que

$$
\left.P^{\prime \prime}(t) P(t)-\frac{3}{2} P^{\prime 2}(t) \geq P^{\prime \prime}(t) P(t)-\frac{(\rho+3)}{2} P{ }^{2}(t)>0 \text { en }\right] t_{0},+\infty[
$$

(recordar que $\rho \geq 0$ ).

Por tanto

$$
\begin{aligned}
& \left.F^{\prime \prime}(t)<0 \text { en }\right] t_{0},+\infty[ \\
& \left.F^{\prime}(t)<0 \text { en }\right] t_{0},+\infty[
\end{aligned}
$$

$F(t)$ es entonces decreciente y concava hacia abajo en $] t_{0},+\infty\left[\right.$ entonces existe $t_{0}$ tal que:

$$
F(t) \rightarrow 0 \text { cuando } t \rightarrow T_{0}^{-}
$$

Es decir

$$
[u(t), v(t)] \rightarrow+\infty \quad \text { cuando } \quad t \rightarrow T_{0}^{-}
$$


Es decir desarrolla una singularidad en tiempo finito en el sentido de la norma, lo cual contradice la suposición inicial de que $T_{\max }=+\infty$, probándose así el teorema.

\section{REFERENCIAS}

1. BAINOV-MINCHEV, E.; Above estimate of the interval of existence of solutions of the nonlinear Kirchhoff equations. Computers Math. Appl. Vol. 31, Nº 2; pp. $63-65$ (1996).

2. CARRILLO, L.E.: On Blow-up for nonlinear Kirchhoff equation. $42^{\circ}$ SBA. UEM Brazil (1995).

3. IKEHATA - OKAZAWA : A class of second order quasilinear evolution equation. Journal of Differential Equations, 114 (1994).

4. LEVINE, H.A.: Instability and nonexistence of Global Solution to Nonlinear wave Equations of the form $P u_{t t}=\Delta u+F(u)$, trans. Amer. Marth. Soc. 192 pp. 1-21 (1974).

5. LIONS, JL.: Quelques Méthodes de Resolution des aux limites non Lineaires. Dunod, París, (1969).

6. MILLA MIRANDA - MEDEIROS, L.A.: On the existence of Global solutions of a Couppled nonlinear Klein - Gordon equations. Funkcialaj Ekvaciaj, Vol. 30, Nº 1. pp. 147-161 (1987).

7. ONO, K. Global Existence, Decay, and Blow-up of Solutions for Some Mildly Degenerate Nonlinear Kirchhoff strings. Journal of Differential Equations 137, pp. 237-301 (1997)

8. SATTINGER, D. H. : On global solutions of nonlinear hyperbolic equation, Arch. Rat. Mech. Anal. 30 pp. 148-172 (1968).

9. TARTAR, L. Topics in Nonlinear Analysis; Univ. De París Sud, Publication D'orsay, France, (1978). 